Research Journal of Applied Sciences, Engineering and Technology 7(1): 30-36, 2014

ISSN: 2040-7459; e-ISSN: 2040-7467

(C) Maxwell Scientific Organization, 2014

Submitted: January 25, 2013

Accepted: March 02, 2013

Published: January 01, 2014

\title{
Fuzzy Control Strategy of Battery Management for PHEV during Regenerative Braking
}

\author{
${ }^{1,}$ Zhumu Fu, ${ }^{1}$ Bin Wang, ${ }^{3}$ Aiyun Gao and ${ }^{1}$ Pengge Zhou \\ ${ }^{1}$ Electronic and Information Engineering College, Henan University of Science and \\ Technology, Luoyang 471023, P.R. China \\ ${ }^{2}$ School of Control Science and Engineering, Shandong University, Jinan 250061, P.R. China \\ ${ }^{3}$ Vehicle and Motive Power Engineering College, Henan University of Science and \\ Technology, Luoyang 471023, P.R. China
}

\begin{abstract}
Based on analyzing the structure of Parallel Hybrid Electric Vehicle (PHEV) and its operation during regenerative braking, a fuzzy control strategy of battery management is proposed. Firstly, the state of charging is estimated by establishing the mathematical relationship between open circuit voltage and the internal resistance model. Secondly, the fuzzy logic controller is designed in regenerative braking system. Finally, by modeling and simulation in ADVISOR, it is shown that the rate of energy recovery with the fuzzy control strategy is increased by 12.3, 18.3 and $7.6 \%$, respectively in three different driving cycles, compared with the benchmark control strategy in the same driving cycles.
\end{abstract}

Keywords: Battery management, fuzzy control strategy, PHEV, regenerative braking

\section{INTRODUCTION}

Battery management is an important subject for Hybrid Electric Vehicle (HEV) (Li et al., 2011). To improve the efficiency of battery management for PHEV during regenerative braking, it is crucial to manage the State of Charge (SOC) of the battery (Yan et al., 2011; Yang et al., 2009; Wirasingha and Emadi, 2011). Some breakthroughs have been made worldwide in the field of battery SOC management (Yang et al., 2009; Sortomme et al., 2011). Many control strategies maintain the SOC within a defined range to reduce energy loss when charging or discharging the battery, such as the online battery management (Adhikari et al., 2010), rule-based and fuzzy logic global control strategy (Ahmed and Cui, 2012) and the control techniques based on driving cycles (Montazeri-Gh and Asadi, 2011). However, the first and the second control strategies need a complex calculation and the last control strategy needs to predict the driving cycles accurately. On the other hand, regenerative braking power is directly related to charging power of battery (Marc et al., 2011; Perez et al., 2006), there are many research results in the area of battery fast charging to obtain more recovery energy during charging (Yan et al., 2011; Diaz et al., 2004). To some degree, fast charging is very useful to improve the SOC. However, if the regenerative braking power is low enough, then the fast charging is a failure. However, most researches focus on battery management without the characteristics of the HEV during regenerative braking, so they don't suit the actual application for PHEV.
Battery management for PHEV during regenerative braking can be regarded as an energy management of PHEV (Adhikari et al., 2010). Fuzzy logic control strategies have been successful to achieve the overall efficiency in energy management system of PHEV (Ahmed and Cui, 2012; Montazeri-Gh and Asadi, 2011; Syed et al., 2009; Abdelsalam and Cui, 2012). To achieve the overall efficiency in regenerative braking system, it needs make a decision on the power split between the battery and the Internal Combustion Engine (ICE) (Li et al., 2011). Therefore, we propose a strategy by considering the characteristics of PHEV during Regenerative Braking with a Fuzzy Control strategy (RBFLC). The RBFLC is designed with two inputs and one output to manage the ICE output to provide charging power to battery. Finally, the simulation model is established in ADVISOR, the performances with RBFLC and in three drive cycles are compared with the ones controlled by the benchmark control strategy in the same drive conditions. Results clearly demonstrate that the RBFLC can significantly improve the energy recovery.

\section{BENCHMARK CONTROL STRATEGY OF PHEV AND SOC ESTIMATE}

PHEV structure and benchmark control strategy: PHEV has two power sources coupled together mechanically through a mechanical device. The structure of PHEV is shown in Fig. 1. The major advantages of this structure are that: 


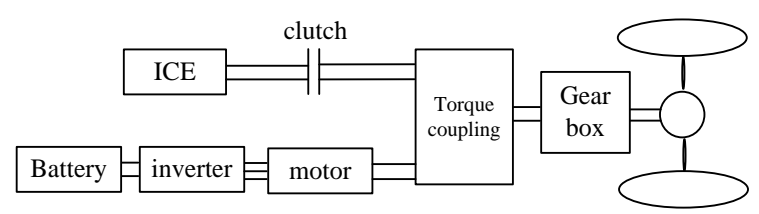

Fig. 1: Structure of parallel hybrid electric vehicle

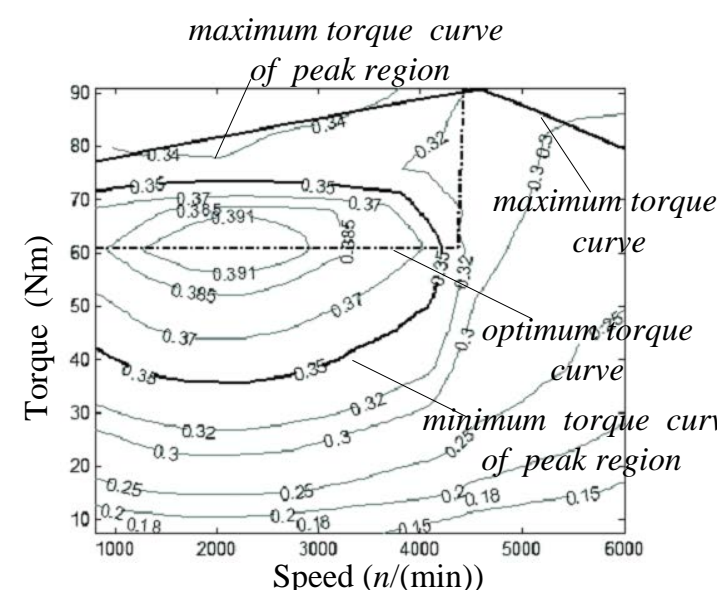

Fig. 2: Peak efficiency region and optimum torque curve of ICE

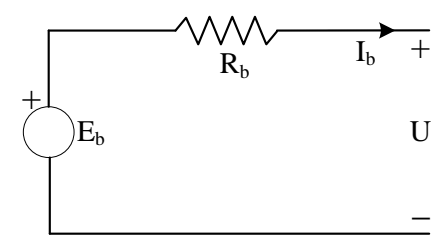

Fig. 3: The equivalent circuit model of battery

- The ICE can transmit its mechanical power directly to the driven wheels

- The ICE can operate in a peak efficient region

The benchmark control strategy is a common control strategy in the application of energy management for PHEV because it is a convenient control strategy. When driving the PHEV, ICE is operating near the optimum torque curve within the peak efficiency region. Both the peak efficiency region and the optimum torque curve of ICE are shown in Fig. 2. The contours show constant efficiencies, which values increase toward inner contours. So the points in dashed line called optimum torque curve are the highest efficiency operating points to ICE at any corresponding speed. We design the peak-efficiency region within the efficiency $\eta_{\text {ice }} \geq 0.35$ to avoid the optimal ICE output torque changing suddenly. The benchmark control strategy defines the maximum and minimum torque curve of peak region used as threshold value to limit ICE output power to the driven wheels, so it is convenient to manage ICE operate within its peak efficiency region by using battery to provide the insufficient power or recycle the redundant power.

However, the benchmark control strategy makes the clutch disconnect during regenerative braking and ICE is useless. In this case, the actual ICE output torque can be expressed as:

$$
T_{\text {ice }}=-T_{\text {inertia }}
$$

where,

$\mathrm{T}_{\text {intertia }}=$ The inertia torque of ICE, $T_{\text {intertia }}=m r^{2} \frac{d \omega}{d t}$

$\mathrm{m} \quad=$ The mass of ICE flywheel

$r \quad=$ The radius of ICE flywheel

$\omega \quad=$ The angular speed of ICE flywheel

When the benchmark control strategy is used, ICE is cut off during regenerative braking, but it has the inertia negative torque and this part of torque is lost. On the other hand, when SOC is low, ICE should provide charging power to battery. However, ICE is also useless. In this case, the SOC may fail to increase quickly and the efficiency of battery charging becomes lower. To make up this defect and make full use of ICE inertia torque, we design a RBFLC which connects the clutch. To achieve the battery management, the SOC of battery should be calculated at first.

SOC estimate: In the process of battery management, a key parameter is the SOC. The SOC of battery is estimated by using an open circuit voltage with the internal resistance model, which consists of an ideal battery with open circuit voltage $E_{b}$ and an equivalent resistance $\mathrm{R}_{\mathrm{b}}$ in series connection. The circuit is shown in Fig. 3.

As shown in Fig. 3, by neglecting the inductance of circuit and assuming charging current hasn't undulating current, the charging voltage $\mathrm{U}$ is expressed as:

$$
U=E_{b}-I_{b} R_{b}
$$

where,

$\mathrm{I}_{\mathrm{b}}=$ Negative current in the charging process

$\mathrm{R}_{\mathrm{b}}=$ Related to the surface temperature of battery $\mathrm{T}_{\text {bat }}$ and SOC, $\mathrm{R}_{\mathrm{b}}=\mathrm{f}\left(\mathrm{T}_{\text {bat }}, \mathrm{SOC}\right)$

According to the relationship among the power, voltage and electric current, an equation is expressed as:

$$
P_{b}=U I_{b}=E_{b} I_{b}-I_{b}^{2} R_{b}
$$

where, $\mathrm{P}_{\mathrm{b}}$ is the power of battery. 
By combining (2) and (3) and noting that $\mathrm{I}_{\mathrm{b}}$ is negative during regenerative braking, $\mathrm{P}_{\mathrm{b}}$ is also negative. $\mathrm{I}_{\mathrm{b}}$ is calculated as:

$$
I_{b}=\frac{E_{b}-\left(E_{b}^{2}-4 R_{b} P_{b}\right)^{1 / 2}}{2 R_{b}}
$$

In the process of charging, SOC is closely related to charging current $\mathrm{I}_{\mathrm{b}}$, on the basis of SOC definition of battery, SOC can be calculated as:

$$
S O C=S O C_{0}+\frac{k_{T_{b}} \int_{0}^{t} I_{b} \eta_{b} d t}{C_{t}}
$$

where, $\mathrm{SOC}_{0}$ is the original $\mathrm{SOC}$ at the beginning of charging and discharging. $\mathrm{k}_{\mathrm{Tb}}$ is temperature coefficient. While ambient temperature is $25^{\circ} \mathrm{C}, \mathrm{k}_{\mathrm{Tb}}=1$. $\eta_{b}$ is the efficiency of charging. $C_{t}$ is the capacity of battery in time $t$.

\section{THE RBFLC SYSTEM DESIGN}

Regenerative braking system design: The schematic of RBFLC system is shown in Fig. 4. FLC uses the regenerative braking torque $\mathrm{T}_{\text {bra }}$ and SOC as inputs and its output is the torque requirement to ICE. However, the FLC output $\mathrm{T}_{\mathrm{FLC}}$ may be up to the maximum requirement torque to ICE, so it should limit the maximum value of demand torque to ICE. The maximum value of demand torque to ICE for PHEV during regenerative braking is calculated as:

$$
T_{\text {dem }}^{\max }=\frac{\left(U_{\max }-E_{b}\right) I_{\max }}{K \omega_{m}}
$$

where,

$T_{\text {dem }}^{\max }=$ The maximum value of demand torque to ICE

$\mathrm{U}_{\max }=$ The maximum acceptable voltage when charging the battery

$\mathrm{I}_{\max }=$ The maximum acceptable charging current, $I_{\max }=\frac{U_{\max }-E_{b}}{R_{b}}$

$\omega_{\mathrm{m}} \quad=$ The angular speed of motor

$\mathrm{K}=$ The gear ratio of motor and ICE

By using $\mathrm{T}_{\mathrm{FLC}}$ and $T_{d e m}^{\max }$, we can calculate the actual demand torque to ICE, the equation is represented as follows:

$$
T_{d e m}^{a c t}=\min \left(T_{F L C}, T_{d e m}^{\max }\right)
$$

where, $T_{d e m}^{a c t}$ is the actual demand torque to ICE.

The module of charging torque provided by ICE calculates the actual output torque of ICE. Then the charging power can be obtained by:

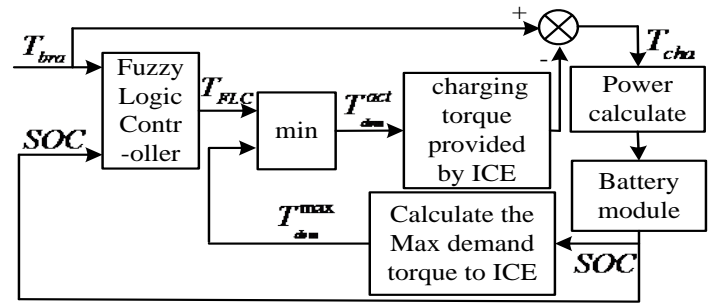

Fig. 4: The schematic of RBFLC system

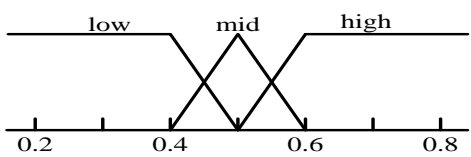

(a) The membership function of SOC

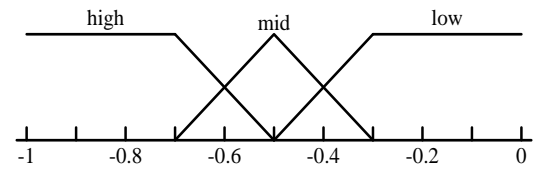

(b) The membership function of $\mathrm{T}_{\text {bra }}$

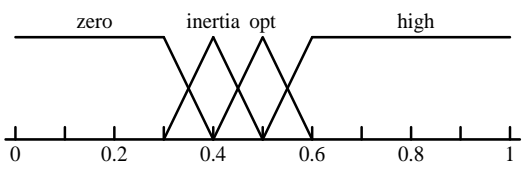

(c) The membership function of $\mathrm{T}_{\mathrm{FLC}}$

Fig. 5: The membership function of inputs and output

$$
P_{\text {cha }}=T_{\text {cha }} \omega_{m}
$$

where,

$\mathrm{P}_{\text {cha }}$ : The charging power from regenerative braking system, $\mathrm{P}_{\text {cha }}=\mathrm{P}_{\mathrm{b}}$

$\mathrm{T}_{\text {cha }}$ : The torque corresponds to $\mathrm{P}_{\text {cha }}, \mathrm{T}_{\text {cha }}=\mathrm{T}_{\text {bra }}-$ $\mathrm{T}_{\text {dem }}^{\text {act }} / \mathrm{K}$

The battery module estimates the SOC, since we know the open circuit voltage $E_{b}$ and the battery charging power $\mathrm{P}_{\mathrm{b}}=\mathrm{P}_{\text {cha }}$, we can calculate $\mathrm{I}_{\mathrm{b}}$ from Eq. (4) and then SOC can be also obtained from Eq. (5).

Fuzzy logic controller design: Based on the above analysis, this study designs a fuzzy controller with two inputs and one output in regenerative braking system. The Mamdani algorithm is used as the reasoning method.

Design the number of the linguistic variables: The more the number of the designed linguistic variables is, the more precise the fuzzy controller is. However, at the same time, the calculation would increase. Therefore, the corresponding linguistic variables are low, mid (middle), high, opt (optimum), inertia (inertial torque), zero. 
Res. J. App. Sci. Eng. Technol., 7(1): 30-36, 2014

Table 1: The rule of fuzzy control

\begin{tabular}{llll}
\hline & Regenerative braking torque & \\
State of Charge (SOC) & High & Mid & Low \\
\hline Low & Inertia & Opt & High \\
Mid & Inertia & Opt & Opt \\
High & Zero & Zero & Inertia \\
\hline
\end{tabular}

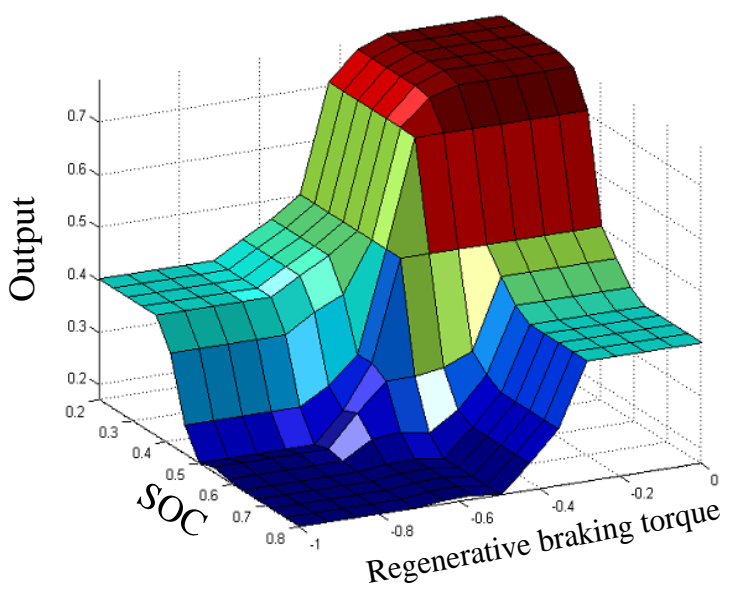

Fig. 6: Control surface

Design the membership functions: The membership functions of the inputs and output are designed as Fig. 5. They use two opening trapezoid membership functions in the two sides and use triangle membership functions in the middle. The range of SOC should be in (0.2 0.8), the minimum SOC value of battery should be controlled above 0.2 , if the SOC is lower than 0.2, RBFLC must provide charging power to battery. The middle value of SOC is 0.5 and charging should be prohibited while the SOC value is up to 0.8 . Since the power flow from ICE or motor to the wheels is defined as positive and the reverse is defined as negative. So the quantized range of regenerative braking torque is $(-10)$ and high is corresponding to the absolute value higher than 0.5 , the middle absolute value is $(0.30 .7)$, low is corresponding to the absolute value lower than 0.5.

The FLC output has four linguistic variables, which corresponds to the ICE output by multiplying quantization factors. For example, the range of linguistic variable opt stays relevant to ICE peak efficiency region. Notice that the linguistic variable inertia, the inertial torque of ICE could be used in two situations:

- $\quad$ SOC is less than or equal to mid but braking torque is high

- SOC is high but braking torque is low

After the design of the membership function, the rules table is designed which would be modified by observing the surface determined by the input and
Table 2: Parameters of vehicle

\begin{tabular}{lll}
\hline Component & Parameter & Value \\
\hline ICE (honda-insight) & Peak power & $50 \mathrm{kw}$ \\
& Optimum torque & $60 \mathrm{Nm}$ \\
& Peak efficiency & 0.4 \\
Motor (insight) & Peak power & $30 \mathrm{kw}$ \\
& Peak torque & $\pm 150 \mathrm{Nm}$ \\
NI-HM battery & Open circuit voltage & $288 \mathrm{~V}$ \\
& Capacity & $6.5 \mathrm{Ah}$ \\
PHEV data & Radius of wheel & $0.275 \mathrm{~m}$ \\
& Frontal area & $1.92 \mathrm{~m}^{2}$ \\
& Total mass & $1150 \mathrm{Kg}$ \\
\hline
\end{tabular}

output variables. The rule of fuzzy control is shown in Table 1.

The Control surface is shown in Fig. 6. We can make conclusions by observing the surface: the lower SOC value of the battery and the regenerative braking torque are, the higher the fuzzy controller output is. On the contrary, the higher the regenerative braking torque and the higher SOC values are, the lower fuzzy controller output is, until it is zero.

In this study, through experiences and simulations, the logical AND has been implemented with the minimum operator, the implication method is minimum, the aggregation method is maximum and the defuzzification method is centeroid.

\section{SIMULATION AND COMPARATIVE ANALYSIS}

To confirm the fuzzy control strategy of battery management for PHEV during regenerative braking, we design the simulation model with fuzzy logic controller in MATLAB/ADVISOR. The important parameters of ISG-PHEV are listed in Table 2. Simulations are carried out in three driving cycles: New Europe Driving Cycle (CYC-NEDC), Urban Dynamometer Driving Schedule (CYC-UDDS) and Japanese 1015 mode driving Cycle (CYC-1015). These cycles are chosen to reflect different driving characteristics. Figure 7 illustrates three of the selected driving cycles.

When the initial SOC of the battery is set to 0.7, the ICE performances with RBFLC are illustrated by Fig. 8, the simulation results of SOC are shown in Fig. 9a-c (Dashed lines correspond to RBFLC, solid lines correspond to benchmark control strategy). We can see ICE is operating in its peak efficiency region and near its optimum curve. Some ICE output torque points operate above the optimum curve because the deceleration or inertial torque of ICE. However, these inertial torque points can provide charging power to battery when RBFLC is used. The advantage of RBFLC is more obvious when PHEV is operating in UDDS. Since there are many times of start-stop state, many output torque points operate above the optimum curve. When using RBFLC, all these points can provide charging power to battery, so the energies loss of ICE is reduced. Respectively, when using the fuzzy logic control strategy, the final SOC is $0.628,0.645$ and 
Res. J. App. Sci. Eng. Technol., 7(1): 30-36, 2014

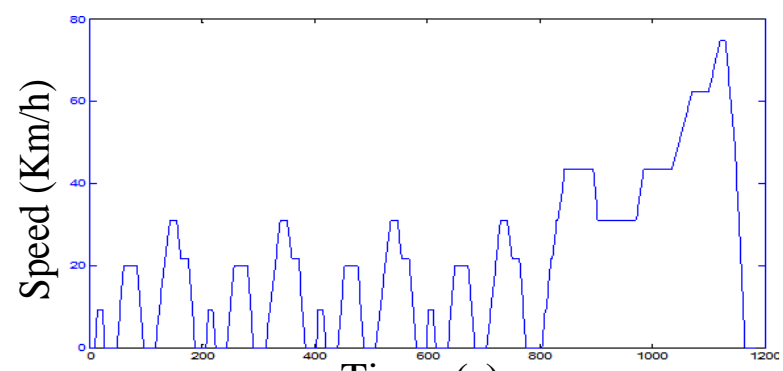

Time (s)

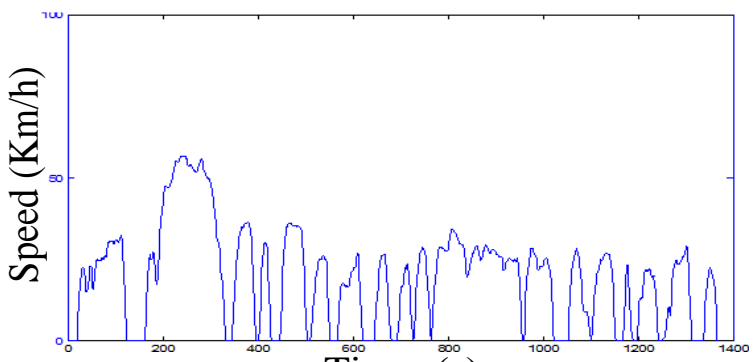

Time (s)

(a) CYC-NEDC

(b) CYC-UDDS

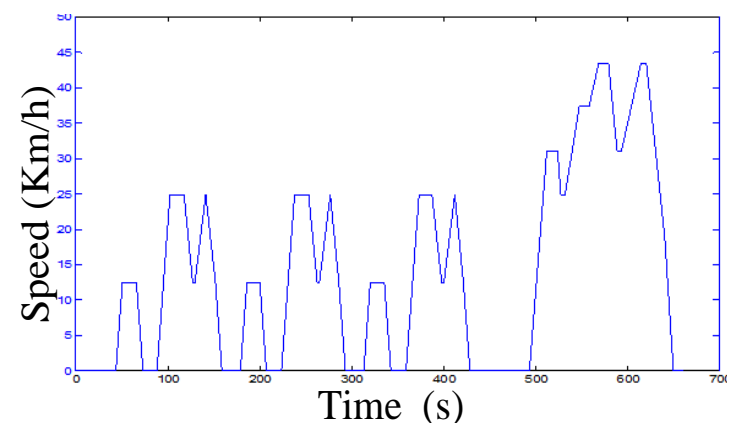

(c) CYC-1015

Fig. 7: Driving cycles

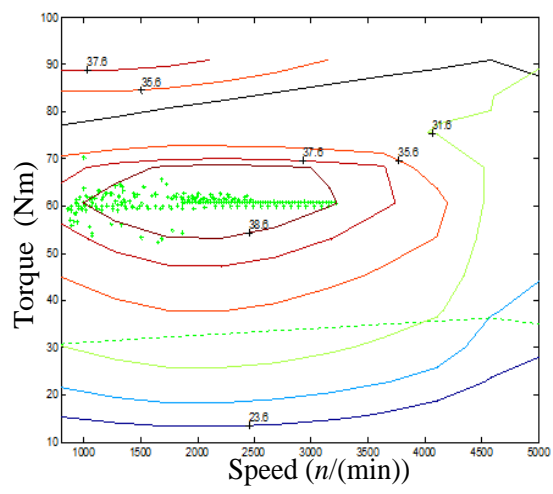

(a) ICE performance in CYC-NEDC

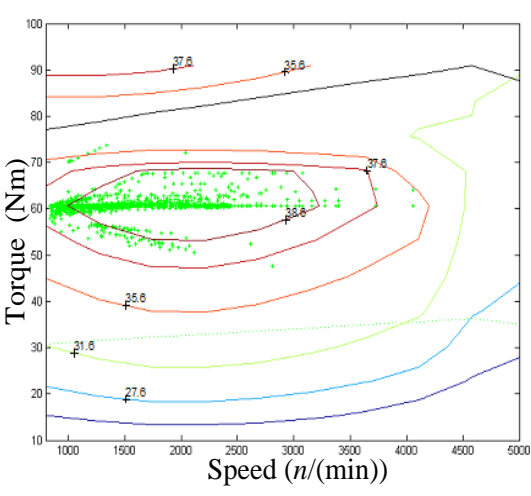

(b) ICE performance in CYC-NEDC

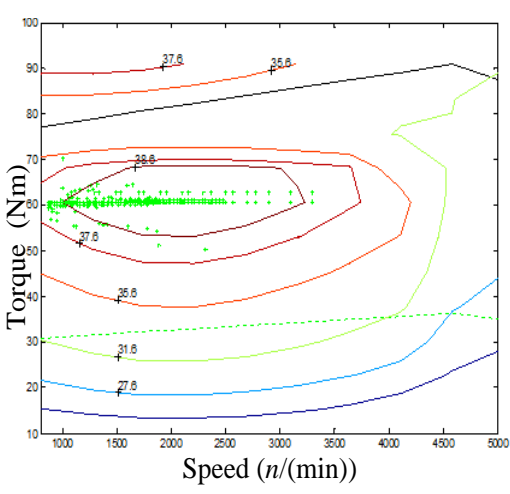

(c) ICE performance in CYC-1015

Fig. 8: ICE performances in three driving cycles

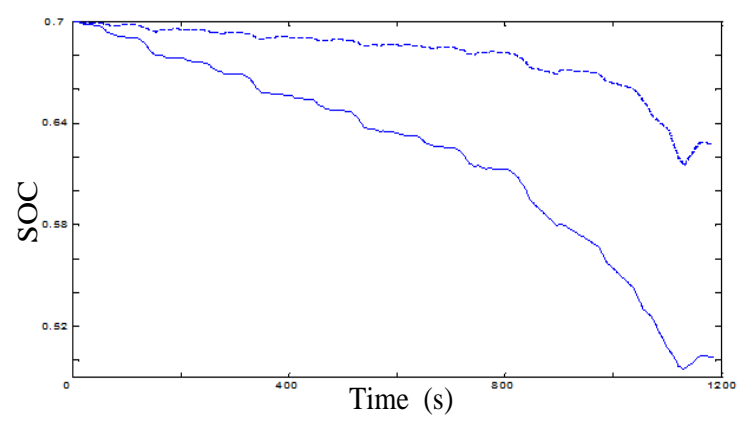

(a)

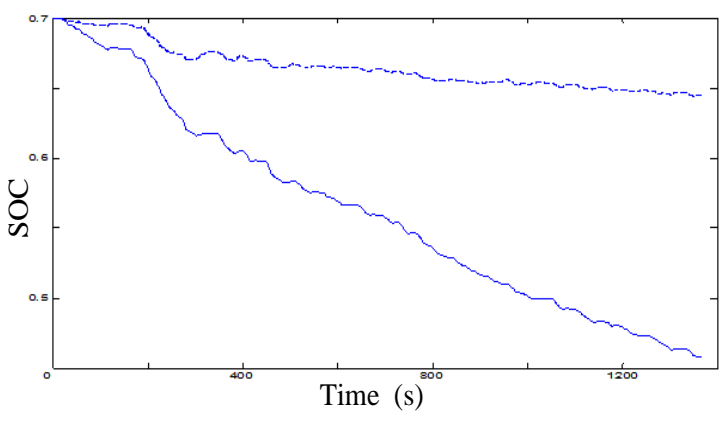

(b) 
Res. J. App. Sci. Eng. Technol., 7(1): 30-36, 2014

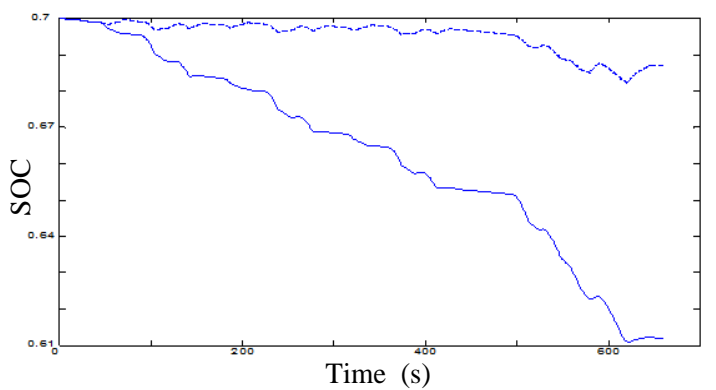

(c)

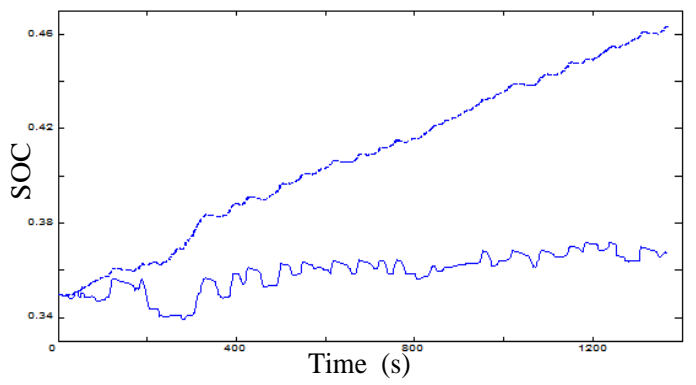

(e)

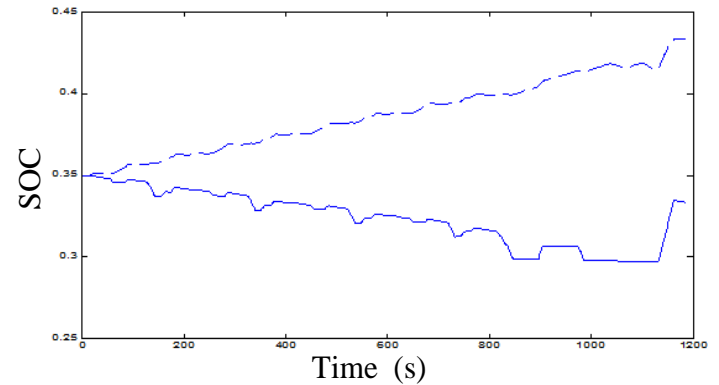

(d)

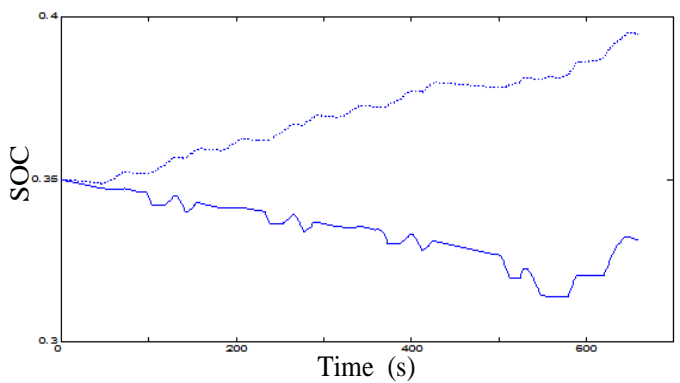

(f)

Fig. 9: The variation of SOC comparison, (a)-(c) the variation of SOC comparison in CYC-NEDC, CYC-UDDS and CYC-1015 when the initial SOC is set to 0.7, (d)-(f) the variation of SOC comparison in CYC-NEDC, CYC-UDDS and CYC-1015 when the initial SOC is set to 0.35

0.688 corresponding to CYC-NEDC and CYC-UDDS, CYC-1015, respectively. Conversely, when using the benchmark control strategy, the corresponding final SOC is $0.505,0.462$ and 0.612 . In other words, the energy of battery with RBFLC is increased by 12.3 , 18.3 and $7.6 \%$, respectively in the three different driving cycles, compared with the benchmark control strategy.

To confirm the fuzzy control strategy of battery management when the SOC is low, the initial SOC is set to 0.35 , the simulation results of SOC are shown in Fig. 9d-f. When we use the fuzzy logic control strategy, the final SOC is up to the initial SOC. It can be seen the more time of driving cycle takes, the higher of the final SOC is. However, the final SOC is very low when the benchmark control strategy is used and the final SOC is increased only in CYC-UDDS.

\section{CONCLUSION}

This study designs a fuzzy control strategy of battery management for PHEV during regenerative braking. The RBFLC uses regenerative braking torque and SOC as input, distributes the power between battery and ICE on the basis of reducing the ICE energy loss and improving the storage energy of battery when SOC is low. Simulation results show that the rate of battery energy with fuzzy control strategy is increased by the energy of battery is increased by 12.3, 18.3 and
7.6\%, respectively in CYC-NEDC, CYC-UDDS and CYC-1015, compared with the benchmark control strategy, when the SOC is low enough, the RBFLC provide more charging power to battery during regenerative, greatly improving recovery energy and proving the high efficiency and reasonable of fuzzy control strategy.

However, the driving cycle is very complex and diverse. Therefore, in the practical application, combining other intelligent technologies with fuzzy control strategy designed for battery management and overcoming the dynamics disturbance is the focus of the future study.

\section{ACKNOWLEDGMENT}

The authors would like to thank the anonymous reviewers for their constructive and insightful comments for further improving the quality of this note. This study was supported by National Natural Science Foundation of P.R. China under Grant 60904023 and Grant No. 51277116.

\section{REFERENCES}

Abdelsalam, A.A. and S.M. Cui, 2012. A fuzzy logic global power management strategy for hybrid electric vehicles based on a permanent magnet electric variable transmission. Energies, 5(4): 1175-1198. 
Adhikari, S., S.K. Halgamuge and H. Watson, 2010. An online power-balancing strategy for a parallel hybrid electric vehicle assisted by an integrated starter generator. IEEE Trans. Vehicul. Technol., 59(6): 2689-2699.

Ahmed, A. and S. Cui, 2012. Different architectures and modes of operation of HEV based on permanent magnet-electric variable transmission with rule-based and fuzzy logic global control strategy. Int. J. Elec. Hybrid Vehicl., 4(1): 69-92.

Diaz, J., J.A. Martin-Ramos, A.M. Pernia, F. Nuno and F.F. Linera, 2004. Intelligent and universal fast charger for Ni-Cd and Ni-MH batteries in portable applications. IEEE Trans. Ind. Elec., 51(4): 857-863.

Li, S.G., S.M. Sharkh, F.C. Walsh and C.N Zhang, 2011. Energy and battery management of a plug-in series hybrid electric vehicle using fuzzy logic. IEEE Trans. Vehicul. Technol., 60(8): 3571-3585.

Marc, D.D., K. Thomas, A. Vincent and F. Michael, 2011. Impacts of an electric powertrain on the breaking sysytem. Auto Technol., 11(4): 28-33.

Montazeri-Gh, M. and M. Asadi, 2011. Intelligent approach for parallel HEV control strategy based on driving cycles. Int. J. Syst. Sci., 42(2): 287-302.
Perez, L.V., G.R. Bossio, D. Moitre and G.O. García, 2006. Optimization of power management in a hybrid electric vehicle using dynamic programming. Math. Comp. Simul., 73(4): 244-254.

Sortomme, E., M.M. Hindi, S.D.J. MacPherson and S.S. Venkata, 2011. Coordinated charging of plugin hybrid electric vehicles to minimize distribution system losses. IEEE Trans. Smart Grid, 2(1): 186-193.

Syed, F.U., M.L. Kuang, M. Smith, S. Okubo and H. Ying, 2009. Fuzzy gain-scheduling proportional-integral control for improving engine power and speed behavior in a hybrid electric vehicle. IEEE Trans. Vehicul. Technol., 58(1): 69-84.

Wirasingha, S.G. and A. Emadi, 2011. Classification and review of control strategies for plug-in hybrid electric vehicles. IEEE Trans. Vehicul. Technol., 60(1): 111-122.

Yan, J.Y., G.Q. Xu, H.H. Qian, Y.S. Xu and Z.B. Song, 2011. Model predictive control-based fast charging for vehicular batteries. Energies, 4(8): 1178-1196.

Yang, M.J., H.L. Jhou, B.Y. Ma and K.K. Shyu, 2009. A cost-effective method of electric brake with energy regeneration for electric vehicles. IEEE Trans. Ind. Elec., 56(6): 2203-2212. 